\title{
SIMULACIÓN DE UNA INSTALACIÓN DE UN PROCESO INDUSTRIAL. PLC, ROBOT E IHM, MEDIANTE OPC
}

\author{
Rocío Pérez Fernández, rociopf91@gmail.com, David Rebordinos d.rebordinos@,vimasl.es
}

Eduardo J. Moya de la Torre, F. Javier García Ruiz, Alfonso Poncela

ITAP. EII, Paseo del Cauce 59, Valladolid, 47011, España. \{edumoy,javgar, poncela\}@eii.uva.es

\begin{abstract}
El objetivo del presente artículo es presentar una instalación de un proceso industrial y la simulación del mismo que sirva como plataforma de prácticas para los alumnos del Master de Informática Industrial. Para ello se diseña una isla robotizada con: tres cintas transportadoras, cinco topes y un robot. Para programar el funcionamiento de la isla y la simulación de su comportamiento se dispone de STEP 7 y TIA Portal para la programación del PLC $y$ del simulador respectivamente y del IHM del operador

El simulador posee las opciones para configurar el tipo de pieza, si es pieza buena o mala, y el código de la misma para controlar si damos dos, tres o cuatro taladros. Se puede iniciar el ciclo completo de simulación o simular las presencias de los elementos y los defectos posibles de la instalación.

A través del IHM se puede observar el estado del ciclo automático, el número de piezas no trabajadas, buenas o malas, configurar el número de ciclo de cada pieza con su trabajo, visualizar los defectos entre otras opciones.
\end{abstract}

Palabras Clave: Simulación, OPC, Step 7, TIA Portal, RobotStudio, ABB, Siemens.

\section{INTRODUCCIÓN}

La simulación consiste en imitar o fingir la realización de ciertas actividades, aunque realmente no se estén llevando acabo, basado principalmente en la tecnología de los ordenadores, por ello, los continuos avances informáticos y tecnológicos de los elementos que los componen, hace que los sistemas de simulación sean cada día más potentes y rápidos, así como más fáciles de programar por personas que no tengan un gran conocimiento sobre teste tema.

En el ámbito de la industria también existe la simulación de procesos industriales que más adelante estarán implantados en la línea de producción, para los cual los fabricantes poseen varias herramientas para poder simular procesos completos, el funcionamiento de sus dispositivos y de programarles sin la necesidad de tenerle físicamente, como, VirtualPlant, PlantSimulation, RobotStudio Factory I/O, SafeConfg, Flexsim, LabView entre otros.
Con todo esto se consigue un aumento de la productividad, de la calidad de programación de la instalación y que se reduzca el coste de puesta en funcionamiento de una instalación.

En el ámbito de la educación, la simulación tiene el atractivo de poder utilizar las diversas herramientas para enseñar a los alumnos a programar y comprobar el buen o mal funcionamiento de lo programado.

Los objetivos planteados en este artículo son:

- Conectar las herramientas de programación más habituales y más utilizadas en la industria para la realización de una instalación completa. Estas herramientas tienen su propia interfaz y lenguaje de programación, así como su propia simulación.

- Programar el funcionamiento de la instalación de manera que quede estructurado y sea mantenible en los distintos lenguajes de programación.

- Estudio y desarrollo de los requisitos de un proyecto industrial, que sirva al alumnado.

La programación del sistema se va a realiza en varias partes:

- Step 7 Siemens: se programará el funcionamiento del autómata, que será el maestro dentro de la red de la instalación.

- Rapid RobotStudio ABB: se programa el funcionamiento del robot.

- TIA Portal Siemens: se programa el funcionamiento de la pantalla de operador IHM y se realizará la simulación.

La elección de los programas se ha realizado en función del grado de utilidad en trabajos de industria, con un software que poseen un lenguaje de programación sencillo y simple, que poseen herramientas que facilitan la utilización y entendimiento, y su interfaz es fácilmente comprensible.

Para poder interconectar los distintos simuladores y herramientas de programación se utilizan las siguientes técnicas:

- La conexión entre TIA Portal y RobotStudio se realiza mediante tecnología OPC. TIA Portal se comporta como cliente OPC del servidor OPC de RobotStudio.

- La conexión entre TIA Portal y Step 7 se realiza mediante conexión entre el simulador PLCSim 
que actúa como servidor OPC y TIA Portal que es cliente OPC.

- La conexión con las variables de Step 7 se realiza mediante conexión PLC Proxy como se explica más adelante.

El presente artículo se organiza de la siguiente manera: después de esta breve introducción donde se presenta la justificaci, en la sección 2 se describe la Tecnología OPC utilizada y su conexión con las distintas herramientas, en la sección 3 se muestra el funcionamiento de la isla, en la sección 4 se presenta la simulación del HMI. Finalmente, en las secciones 5 y 6 se muestran los resultados obtenidos y algunas de las principales conclusiones extraídas para su posterior utilización como equipo de prácticas en el Master de Informática Industrial en la Universidad de Valladolid, así como las referencias utilizadas.

\section{Tecnología OPC}

\section{1 ¿Qué es la tecnología OPC?}

Las siglas OPC significan OLE for Process Control, es un estándar de comunicación y de conectividad de datos en el campo de control y supervisión de procesos industriales, la cual utiliza una interfaz común de comunicación con la que se consigue la interconexión e intercambio de datos entre diferentes herramientas software (gestiona OPC Foundation). Esta tecnología se utiliza para evitar el problema de la comunicación de dispositivos, controladores o aplicaciones sin utilizar protocolos propietarios de cada herramienta. Utiliza la arquitectura clienteservidor de manera que el servidor es la fuente de datos y el cliente puede acceder a este servidor para leerlos o escribirlos.

En este tipo de conexión pueden existir varios dispositivos conectados a la vez. En nuestro caso la arquitectura Cliente-Servidor OPC funciona de la siguiente manera:

- Servidor OPC.

Es una aplicación software estandarizada encargada de conectar varios clientes OPC o herramientas software de proveedores diferentes.

Un servidor OPC puede leer y escribir en un cliente OPC, tiene el papel de esclavo, es decir se comporta como el receptor de la información y la trata o la vuelve a transmitir.

\section{- Cliente OPC.}

Es una aplicación software estandarizada encargada de comunicarse con un servidor OPC a través de un tipo de mensajería estandarizada.

El cliente OPC es un sistema donde se tiene los datos que se van a comunicar con el Servidor OPC. El cliente toma el papel de maestro, es el controlador de la comunicación, es el encargado de iniciar y controlar la comunicación con el servidor, le envía unos datos y recibe los devueltos por el Servidor.

En el caso de la aplicación del presente proyecto se tiene la siguiente configuración cliente-servidor.

- Step 7 tiene el simulador PLCSim que trabaja como un servidor OPC.

- RobotStudio se tiene IRC5 Servidor OPC.

- TIA Portal se tiene la simulación de la parte del runtime, que se comporta como cliente servidor.

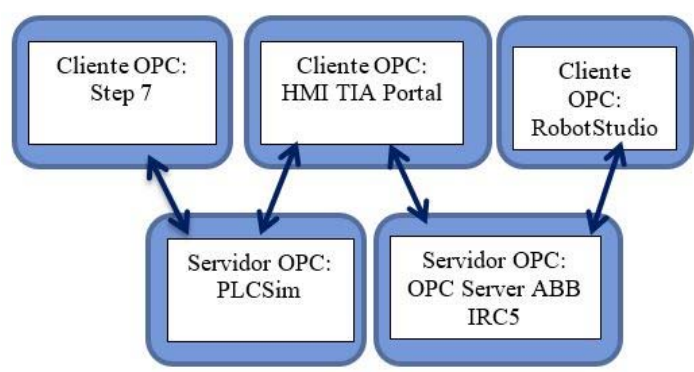

Figura 1: Conexión programas cliente-servidor

\subsubsection{STEP7 - PLCSim}

Arrancando STEP7 se tiene el icono de simulación de PLCSim:

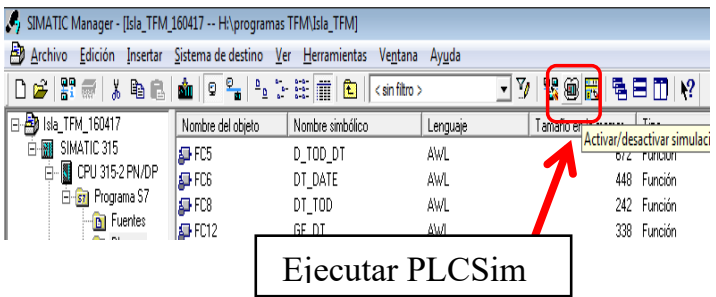

Figura 2: Captura de pantalla icono PLCSim

Pulsando, se abre la pantalla del Servidor OPC de Siemens PLCSim. Para que tenga un correcto funcionamiento se debe configurar la conexión PLCSim TCP/IP y se debe activar el estado del PLC a Run-P.

De esta manera se pueden leer y escribir todas las variables del PLC, sin tener que configurar nada más.

\subsubsection{RobotStudio}

Para configurar el Servidor OPC de RobotStudio hay que realizar varios pasos.

1) Primero se debe activar el sistema ServerOPC de RobotStudio: Se ejecuta el sistema ABB IRC5 OPC Configuration, y se añade un nuevo Alias, es decir una nueva conexión.

2) Se debe encontrar el sistema OPC de RobotStudio, para ello se pulsa el icono Scan.

3) Se buscan las conexiones, y cuando aparece la que interesa se selecciona y se pulsa a crear.

4) Por último, para que se ejecute la conexión se debe arrancar esta, para ello en la pestaña Server Control se pulsa el botón Start. 


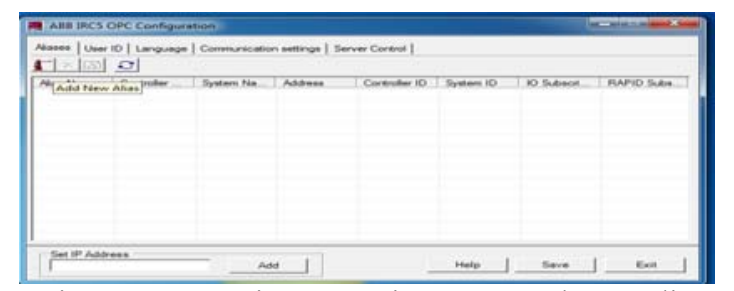

Figura 3: Creación conexión OPC RobotStudio

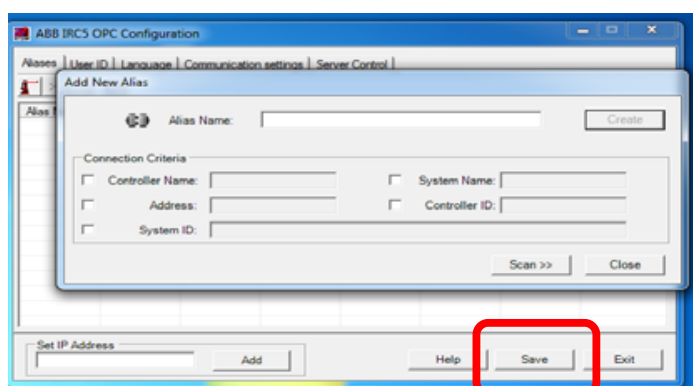

Figura 4: Búsqueda servidores OPC RobotStudio

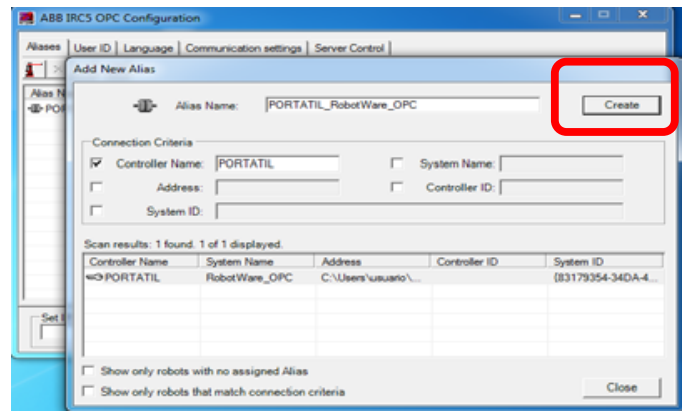

Figura 5: Creación conexión OPC RobotStudio

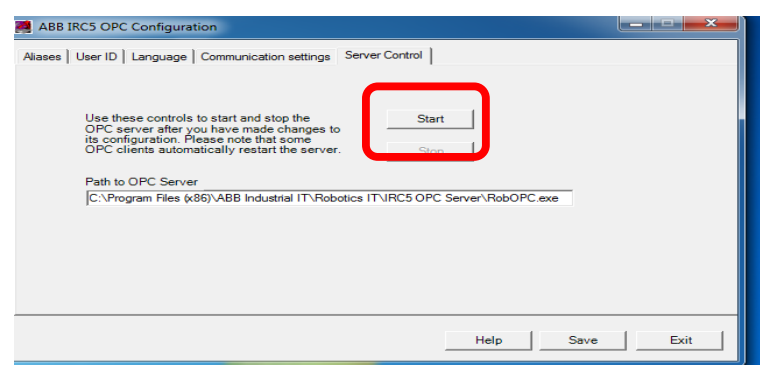

Figura 6: Arrancar OPC RobotStudio

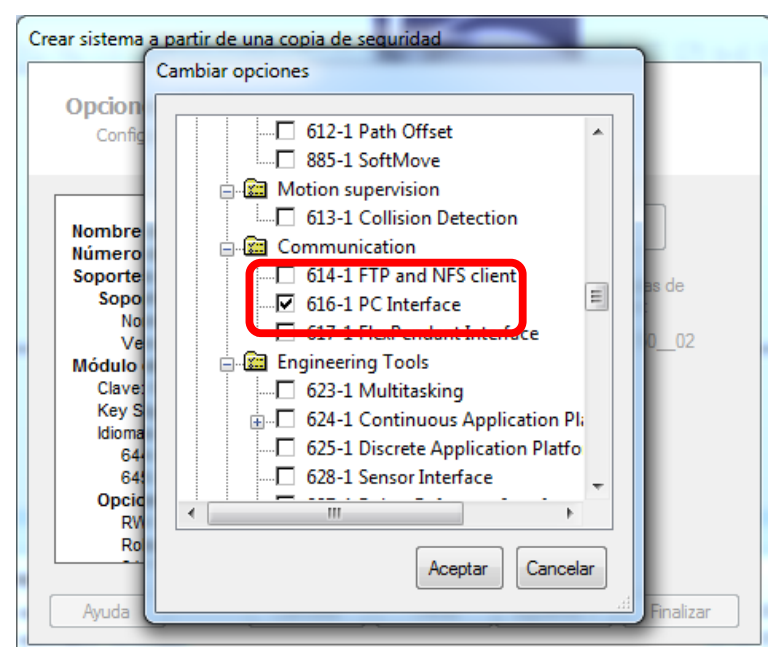

Figura 7: Opción OPC del control de RobotStudio
Una vez ejecutados estos pasos ya se tiene configurado el Servidor OPC de RobotStudio, pero para que se puedan gestionar las variables que se leen por el servidor se debe configurar también la herramienta RobotStudio, y el controlador de manera que posea las comunicaciones OPC, para ello se busca la opción PC-Interface y se activa.

A continuación, se debe determinar las variables que van a ser leídas por el sistema OPC, esto se realiza de la siguiente forma: en la pantalla del controlador, en Entradas/Salidas (I/O) se configura un nuevo grupo de señales que serán las que interaccionen con el sistema OPC

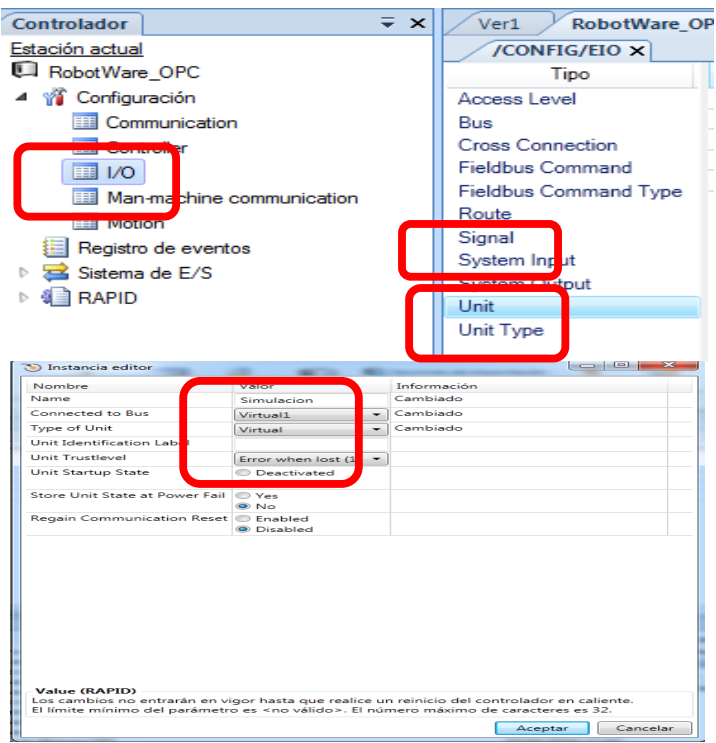

Figura 8: Creación variables simuladas RobotStudio

El último paso es configurar y definir las variables a interconectar, para ello en la parte de Signal se crean las señales que se van a comportar como entradas o salidas. Cuando se creen nuevas variables se tiene que volver a reiniciar el controlador y el servidor OPC antes configurado, de la manera que se realizó su arranque Start anteriormente.

\subsubsection{TIA PORTAL}

Una vez configurada los servidores OPC del sistema se debe interconexionar estos con el cliente OPC, TIA Portal, desde la opción de conexiones. Se crea una nueva conexión con el PLC y otra con el Robot.

\section{a) Para la creación de la conexión PLC}

Una vez configurada los servidores OPC del sistema se debe interconexionar estos con el cliente OPC, TIA Portal, desde la opción de conexiones, creando una nueva conexión con el PLC y otra con el Robot.

\section{b) Para la conexión con el robot}

Se selecciona el tipo de conexión OPC y dentro de la lista de servidores OPC existentes se selecciona el de RobotStudio. 

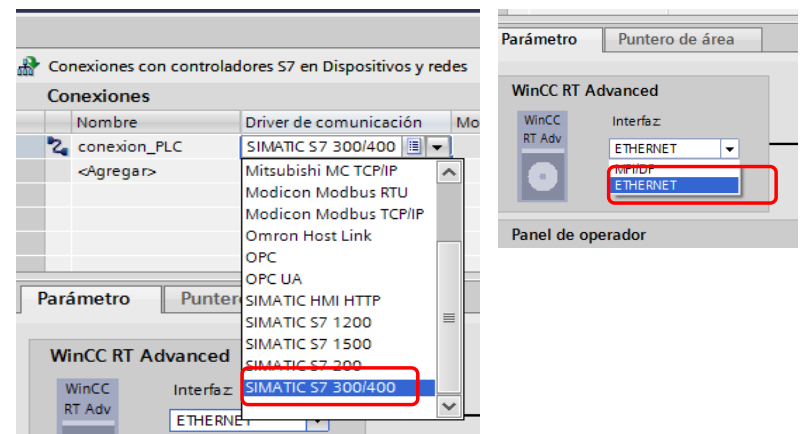

Figura 10: Conexión TIA Portal con PLC

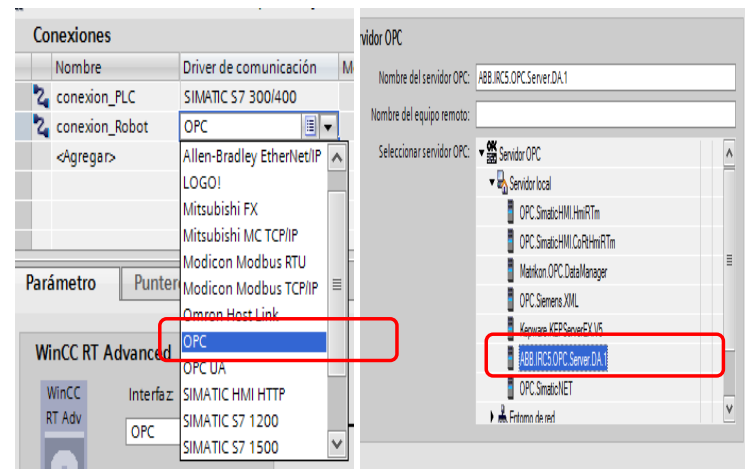

Figura 11: Conexión TIA Portal con RobotStudio

c) Conexión PLC con HMI - Device PLC Proxy

PLC Proxy se utiliza para poder crear un proyecto de autómata con STEP7 y la configuración IHM con el software WinCC TIA Portal, a través de PLC_Proxy se conexionan los dos programas.

Con este servidor se consigue un acceso directo al controlador y a su configuración (variables del proyecto con sus nemónicos, bloques de datos (DB) del proyecto, a los defectos y alarmas, etc.)

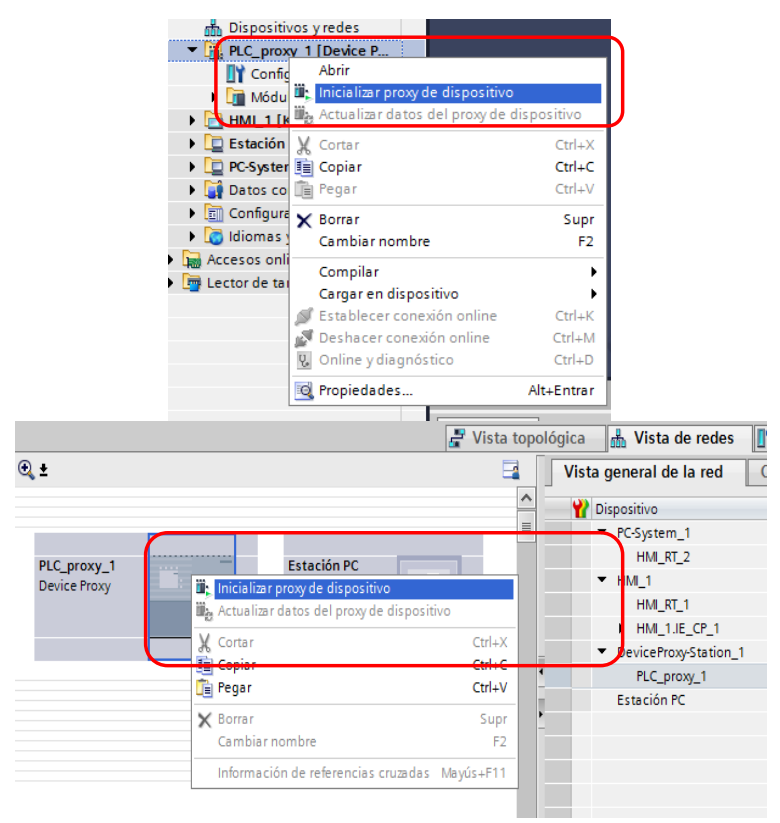

Figura 12: PLC_Proxy

Una vez agregado el dispositivo PLC Proxy se debe conectar el dispositivo con el programa autómata, para ello hay dos opciones: con el botón derecho encima del nombre en el árbol de la izquierda, y dar a inicializar proxy de dispositivo, o en la vista de redes, pinchando en el dispositivo con el botón derecho, en la misma opción inicializar proxy dispositivo.

A continuación, se debe seleccionar el programa autómata de donde se quieren importar las variables que se desean pasar a la simulación y se selecciona por último los datos y variables a añadir al servidor. Terminado de crear todos los elementos se deben conexionar mediante la red Ethernet.

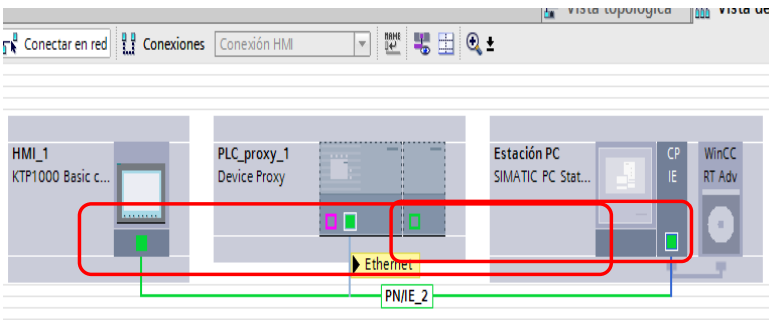

Figura 13: Conexión hardware

\section{FUNCIONAMIENTO DE LA ISLA}

Los requisitos y la descripción del funcionamiento de la Isla es el siguiente:

- La isla consta de un robot (R), tres cintas transportadoras (cinta de entrada $\mathrm{C}$, cinta de salida piezas buenas $\mathrm{C} 2$ y cinta de salida piezas malas C3), cuatro topes (Tope de entrada, tope de la isla, tope salida piezas buenas y tope salida piezas malas).

- La cinta de entrada tiene dos puntos de parada, en la posición dentro de la isla donde se trabaja la pieza y en la posición anterior a la entrada a la isla, donde espera la pieza si existe una dentro.

- Si no hay pieza en el tope de la isla, el PLC autoriza la entrada de la pieza a la isla, por lo tanto, manda la orden de apertura al tope de entrada.

- La pieza avanza hasta el tope de la isla, momento en que el PLC recoge la información del tipo de pieza que se trata. Entre estos datos están los de si se trabaja la pieza o no en esa isla, y si la pieza se trabaja, que tipo de pieza es y que trabajos tiene que realizar.

- Si la pieza no se trabaja en la isla, el PLC manda la orden de apertura al tope de la estación que se abre y deja pasar la pieza hasta el final del transportador.

- Si la pieza si se trabaja, el PLC manda la orden de indexar la pieza, para que cuando se realice los trabajos, ésta siempre se encuentre en la misma posición.

- Cuando el PLC le autoriza a realizar el trabajo, el robot R se posiciona en los puntos donde controla 
los taladros realizados, en función del tipo de pieza.

- Esta información de pieza buena o pieza mala se la envía el PLC al robot.

- Cuando el PLC le dice al robot que puede coger la pieza, éste la coge y le contesta al PLC que tiene cogida la pieza, momento en que el PLC le autoriza que se vaya con la pieza cogida.

- En función del tipo de pieza informado al robot:

$\checkmark$ Si es pieza buena, éste se aproxima al punto de dejada en la cinta transportadora de salida de piezas buenas $\mathrm{C} 2$.

$\checkmark$ Ésta aproximación del robot a la cinta de salida de piezas buenas se realiza siempre y cuando el PLC de la autorización y la información de que no existe pieza en la posición de dejada de la cinta de salida de piezas buenas.

$\checkmark \mathrm{Si}$ es pieza mala, éste se aproxima al punto de dejada en la cinta transportadora de salida de piezas mala $\mathrm{C} 3$. La aproximación del robot a la cinta de salida de piezas malas se realiza siempre y cuando el PLC dé la autorización y la información de que no existe pieza en la posición de dejada de la cinta de salida de piezas malas.

- Una vez el robot se aproxima a la cinta de salida y tiene la autorización de dejar la pieza, éste posiciona la pieza en su lugar e informa al PLC, momento en el que el PLC le debe dar la autorización de retroceso de posición.

\section{SIMULACIÓN MEDIANTE HMI}

El HMI creado consta de las siguientes pantallas:

- PANEL DE CONTROL: Se puede pulsar los distintos controles de la isla como si del panel de control de una instalación real se tratara. Selector de modo automático y modo manual, pulsador de puesta en servicio, pulsador de marcha ciclo, pulsador de anulación de defectos.

- DETECTORES DE PRESENCIAS: Se visualiza el sinóptico de la isla, junto con las entradas de los distintos detectores existentes en ella. Todos estos detectores se pueden seleccionar, de manera que se activen como si se tratara de una detección real.

- ARMARIO DE LA ISLA: Se visualiza el nombre de los disyuntores, los relés...que existen en la isla acompañados de un botón cada uno, de manera que se pueda seleccionar el disyuntor que se desea activar.

- SIMULACIÓN DEFECTOS: Se visualizan algunos de los posibles defectos que puedan aparecer en la isla, los que puedan ser simulados. Cada defecto está acompañado de un botón, de manera que se pueda seleccionar el defecto a simular. Para llevarlo a cabo se debe pulsar a continuación el botón "Forzar"

- SINÓPTICO ISLA: En esta pantalla se visualiza las distintas partes que componen la isla. Se visualizan los movimientos que suceden durante el ciclo, así como el estado de cada componente.

Con la simulación se pretende simular las señales de las entradas del sistema, de manera que se pueda visualizar el funcionamiento del sistema sin tenerlo físicamente. Dentro de cada pantalla descrita anteriormente se tienen los siguientes elementos con la siguiente funcionalidad.

Existen varias opciones que hay que configurar, antes de realizar una simulación completa desde dicha pantalla:

- Pieza buena, pieza mala. Para determinar el resultado del trabajo.

- Código de programa. Campo de entrada para introducir el número de pieza con el que se decodifica los trabajos.

- Crear o no pieza. Para comenzar la creación de una pieza nueva en el ciclo.

Toda la simulación se realiza de la siguiente manera:

- Se tiene el botón de selección, cada uno correspondiente con la entrada específica que acompañe al texto, a través de la pulsación del botón "Forzar" que corresponda con la validación por parte del usuario a realizar lo indicado.

- Este traspaso de información del simulador al autómata se realiza a través de un script en lenguaje Visual Basic de TIA Portal.

- Se determina una matriz, que corresponda a un bloque de datos (DB) del autómata, que corresponda a cada entrada del PLC, de manera que, al activar cada elemento de la matriz, se active la entrada adecuada.

- También se tiene la opción de simular un ciclo completo de la instalación con una pieza.

- Se puede elegir simular una pieza buena o mala y se puede seleccionar si simular una pieza tipo A, tipo $\mathrm{B}$ o tipo $\mathrm{C}$, que se configura a través del IHM.

\subsection{SIMULACIÓN ROBOTSTUDIO}

Además de las pantallas de simulación en TIA Portal, se realiza la simulación conjunta en RobotStudio a través de los componentes inteligentes, y cuyos requisitos son los siguientes:

- La simulación consta de tres transportadores, cuatro topes, un robot, un centrador.

- Las piezas aparecen en la simulación, siempre y cuando se active el botón "Crear Pieza" descrito anteriormente en la pantalla Simulación Sinóptico. 
- El robot una vez fijada la pieza comienza a moverse y a realizar el trabajo.

- El robot retrocede, cuando tiene le permiso de coger la pieza, lo hace y la lleva al transportador que corresponda, si es mala al transportador de la izquierda, y si es buena al transportador de la derecha.

- Una vez el robot se retira a la posición inicial o de origen, el tope se abre y la pieza comienza a deslizarse por el transportador, desapareciendo cuando llega al final de este.

\section{RESULTADOS}

Una vez configurada y programada la isla al completo, para poder probar el funcionamiento $\mathrm{y}$ visualizar los movimientos y trabajos que se van a realizar en la isla, con el simulador del software, hay que seguir los siguientes pasos:

1. Abrir el simulador PLCSim de Step7.

2. Cargar el programa en el simulador.

3. Abrir y activar el ServidorOPC de RobotStudio

4. Abrir el simulador de RobotStudio.

5. Activar la simulación en RobotStudio.

6. Abrir el simulador de la pantalla IHM de TIA Portal.

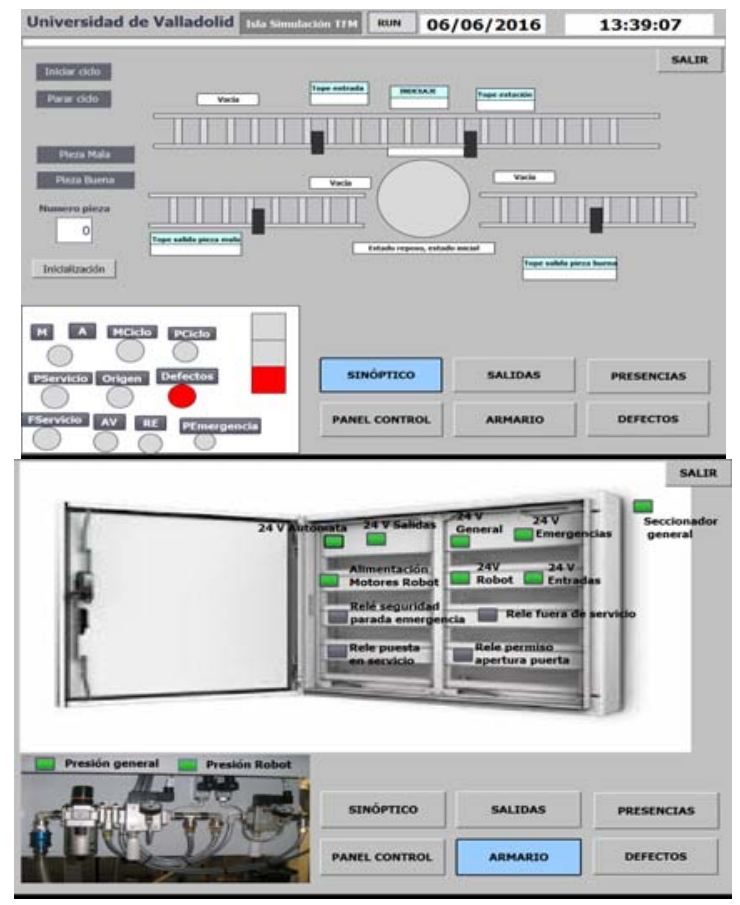

Figura 14: Pantalla Simulador

Para poder activar los ciclos y mover elementos en la instalación se tiene que tener todos los elementos eléctricos correctos y la neumática activada, para ello en el simulador se debe activar todos los magnetotérmicos del armario. En el armario también se pueden forzar los distintos relés existentes y los presostatos del circuito de neumática.

En la simulación del panel de operador se activa la puesta en potencia de la instalación, rearme de relés de seguridad y activación del aire.

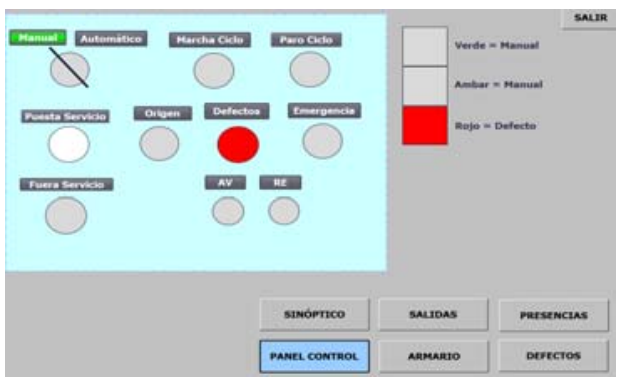

Figura 15: Panel de Control de Simulación

Se pulsa el botón de anulación defectos para anular los defectos que existan en la máquina, si por un casual algún defecto persiste, se puede visualizar el defecto en la pantalla operador.

Una vez activada la electricidad y la neumática de la máquina, se puede inicializar la posición inicial de la instalación o posición de origen.

Para tener la máquina en origen, como inicialmente no están simulados los elementos del campo se debe seleccionar el botón: Inicializar o el botón de origen, de esta manera quedarán los elementos en el estado inicial.

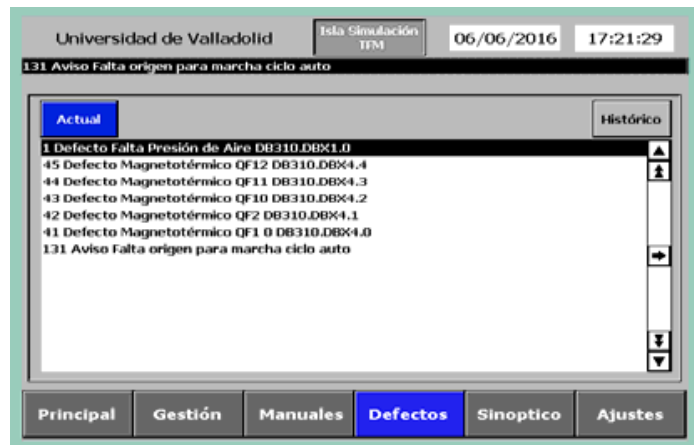

Figura 16: Defectos Panel Operador

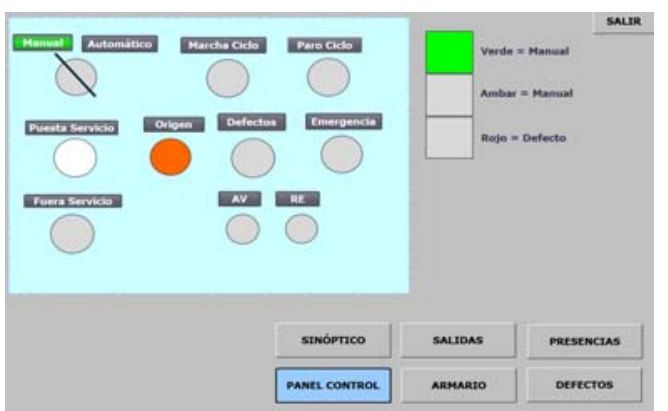

Figura 17: Panel de Control Simulación en marcha

Se selecciona el modo manual en el selector Manu/Auto, dependiendo del modo en que se quiera la máquina, en modo manual se podrán realizar movimientos manuales de algunos elementos de la isla, resetear defectos...etc. En modo automático, siempre y cuando no existan defectos se puede activar el ciclo de la isla. 


\subsection{SIMULACIÓN DE CICLO AUTOMÁTICO}

En la página principal del simulador se pulsa el botón de Iniciar Ciclo, de esta manera comienza a ejecutarse un script donde se van actualizando los valores de los elementos a través de la comunicación OPC con RobotStudio y la pieza va moviéndose a través de la cinta, esto se visualiza tanto en el simulador de TIA Portal como en el de RobotStudio. Antes se debe seleccionar el número de programa de la pieza que se ha configurado en el panel de operador y si la pieza a simular es buena o mala. El número de programa de la pieza se configura en la pantalla del IHM antes de comenzar el ciclo:

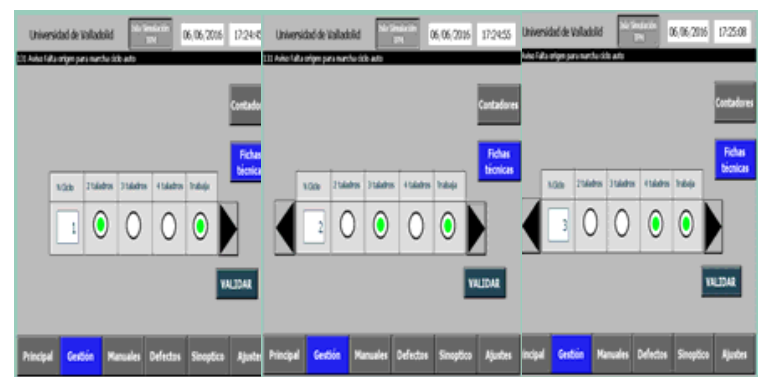

Figura 18: Pantalla configuración ciclo pieza IHM

Una vez configurado el tipo pieza se pasa a simular el proceso. Se selecciona el número 1 de ciclo de programa y pieza buena. Se observa cómo va pasando por los topes, como el robot realiza las tareas correspondientes y una vez finalizadas, posiciona la pieza en su correspondiente cinta de salida, dependiendo de si es pieza buena o mala. La pieza continúa el ciclo y sale por su cinta, correspondiente desapareciendo cuando acaba la misma.

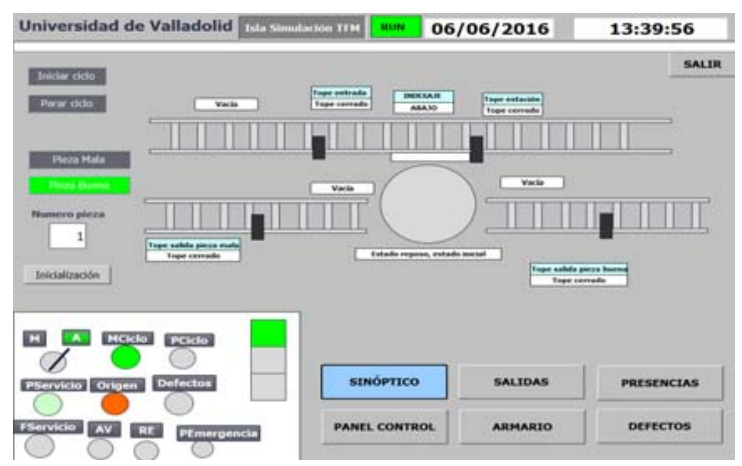

Figura 19: Pantalla sinóptico estado simulación

\subsection{SIMULACIÓN DETECTORES}

Se simulan las posiciones de los detectores físicos que existen en la isla, los topes, indexajes, etc... y la posición de la pieza dentro de la isla. Para ello se pulsa en el botón del HMI SCADA Detectores.

Se debe seleccionar los elementos a modificar su posición y pulsar Forzar Valor para que se cambie el valor de los elementos. Así se puede ir reproduciendo el ciclo forzando el estado de los elementos por el usuario.

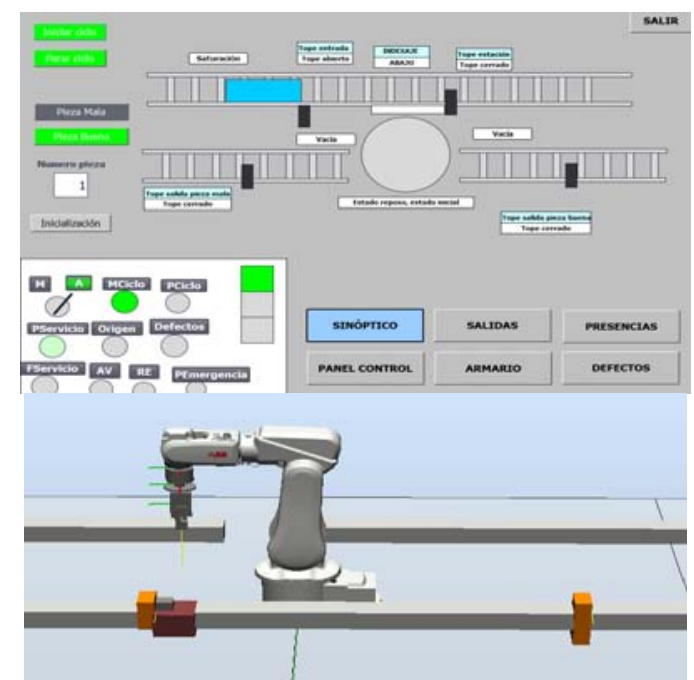

Figura 20: Pantalla simulación estado elementos

Para inicializar los elementos al estado de origen, en la pantalla principal se tiene la opción del botón: Origen o Inicialización. Con esta simulación de detectores también se puede simular un ciclo paso a paso, cambiando en cada ocasión los detectores en el lugar indicado.

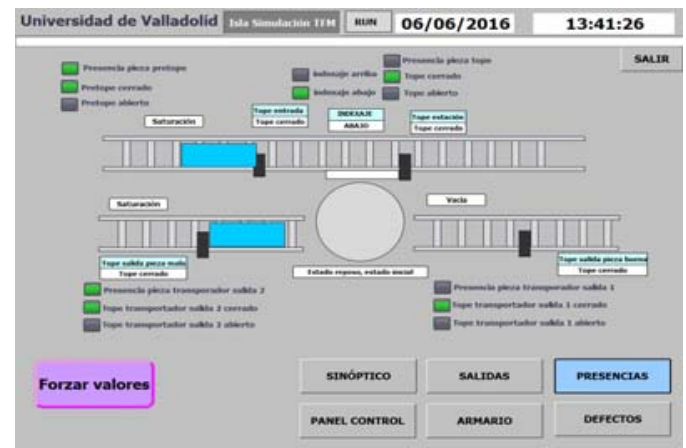

Figura 22: Pantalla simulación detectores

\subsection{SIMULACIÓN DEFECTOS DE LA ISLA.}

Para poder observar que ocurre cuando se produce un defecto, en la pantalla de simulación existe el botón Defectos, dentro del cual hay una lista de los defectos que pueden ocurrir durante su funcionamiento. Para simularlo se debe seleccionar el botón del defecto que se quiere simular y pulsar Forzar Valor. De esta manera se provoca el defecto y se visualiza por la pantalla HMI.

\subsection{SIMULACIÓN PANTALLA OPERADOR.}

Durante la simulación o cuando está en estado de reposo la isla, en la pantalla de operador IHM se puede visualizar el estado de la etapa en la que se encuentra la instalación o el ciclo, el número de piezas que ha pasado por la estación, los defectos existentes o los que han ocurrido en la estación, incluso se puede configurar el tipo de piezas que van 
a pasar por la estación.

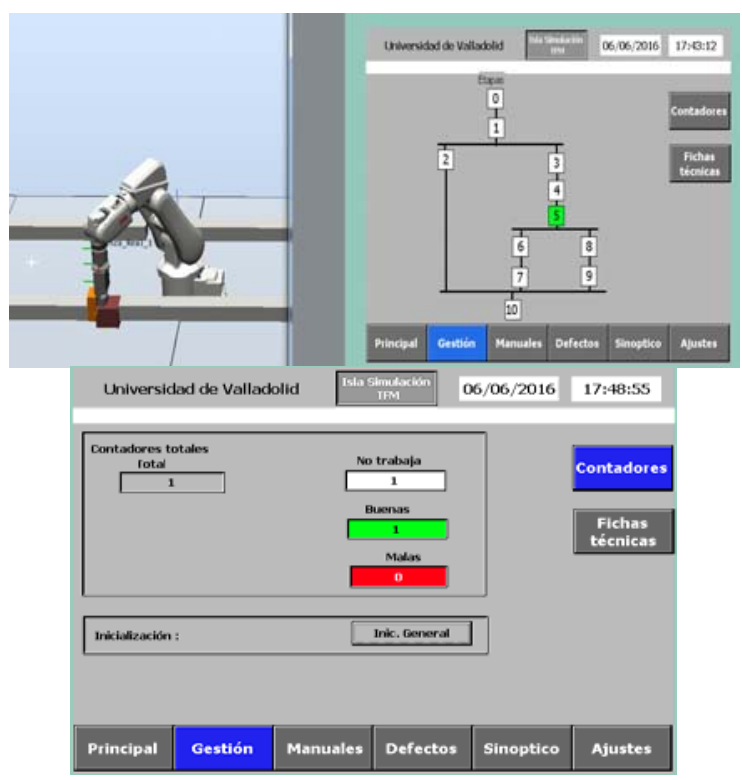

Figura 23: Pantalla IHM operador, estado máquina

\section{CONCLUSIONES}

En definitiva, la finalidad de este artículo es presentar una práctica de laboratorio, de un programa de simulación para observar el funcionamiento de una isla robotizada y de las partes que la componen, de manera que se pueda interactuar con el sistema como si se tratara del sistema real y poder probar el funcionamiento y los posibles defectos y errores tanto de funcionamiento como de programación antes de implementarlo en la isla real.

Tras estudiar y observar los diferentes tipos de softwares que se utilizan en la industria se opta por la utilización y programación del simulador con los programas Step 7 de Siemens, TIA Portal de Siemens y RobotStudio de ABB, ya que en la industria son los programas de autómatas y robots más utilizados. Son programas que poseen lenguajes de programación fáciles y rápidos de aprender. Cuentan también con las ventajas de velocidad, potencia y alcance funcional a la hora de desarrollar este tipo de aplicaciones.

Se ha elaborado los requisitos de cada sistema por separado: funcionamiento de la isla, lo que sería el funcionamiento real de ésta $y$, por otra la simulación del proceso de la isla robotizada.

Se determinó que para crear un simulador lo más real posible, el sistema se tenía que componer de:

- Programa de Autómata: Controla el ciclo de la isla, posee el papel de controlador principal.

- Programa de Pantalla de operario IHM: Panel que existe en la isla real a través del cual el operador puede visualizar los datos y estado de los elementos y realizar movimientos manuales.

- Programa de robot: Realiza los trabajos en las distintas piezas que atraviesan la isla.
Para crear el simulador completo habría que definir un sistema SCADA con lo cual se pudiese visualizar el estado de los elementos reales de la estación, así como poder forzar los valores o estados de estos elementos y poder ejecutar un proceso completo de la isla paso a paso automáticamente.

Se ha elaborado un estudio y la configuración que se debe realizar entre los distintos softwares de manera que se puedan conectar vía OPC para poder realizar la simulación.

Este desarrollo se ha realizado de manera que sea lo más aproximado a la realidad que se encuentra en la industria o en cualquier empresa, y así se entienda el funcionamiento real de una máquina $o$ isla robotizada, y se estructure el desarrollo de un proyecto de automatización y robotización industrial. Al utilizar estos tipos de programas de programación de autómatas y robots, se aprende a manejar este entorno y a desarrollar algoritmos de programación para una aplicación industrial, en unos entornos que, una vez concluido sus estudios el alumno, le sea de utilidad para incorporarse al entorno laboral.

Agradecimientos

Al Proyecto de Innovación Docente PID1718_95

\section{Referencias}

[1] Apuntes de Programación de Entornos Robotizados, Año 2016/2017, UVa. E.I.I. Máster en Informática Industrial.

[2] Apuntes de Programación de Autómatas y SCADAS, Año 2016/2017, UVa. E.I.I. Máster en Informática Industrial.

[3] Apuntes de Simulación de Procesos Industriales, Año 2016/2017, UVa. E.I.I. Máster en Informática Industrial.

[4] Apuntes Ingeniería del Software en Entornos Industriales, Año 2016/2017, UVa. E.I.I. Máster en Informática Industrial.

[5] Apuntes de Gestión de Proyectos Industriales, Año 2016/2017, UVa. E.I.I. Máster en Informática Industrial.

[6] https://www.youtube.com/watch?v=T8YE9S8bHg. Tutorial de RobotStudio (última consulta 7 junio 2017).

[7] https://www.youtube.com/watch?v= gSQcvkKY9HU. Tutorial de RobotStudio (última consulta 7 junio 2017).

[8] https://www.youtube.com/watch?v= rYYVDdOm2QE. Tutorial de conexión OPC RobotStudio. (última consulta 7 junio 2017).

[9] J. A. Avila Herrero, "Modelado de una célula robótica con fines educativos usando el programa Robot-Studio", UVa, 2015.

[10] VIMA S.L. Manuales de Funcionamiento programas Step 7, RobotStudio y TIA Portal. 